\title{
La derivación y el cambio de clases de palabras en el mapudungun*
}

\author{
Elisa Loncon Antileo**
}

\begin{abstract}
Resumen
En este estudio se analiza el proceso de cambio de clases de palabras en el mapudungun a través del procedimiento de derivación, procedimiento de creación de palabra que consiste en un lexema verbal, sustantivo, adjetivo, verbo o adverbio unido a un sufijo derivativo. Por su naturaleza se trata de un estudio descriptivo, que sigue la perspectiva tipológica de la formación de palabra. La hipótesis que se desarrolla es la siguiente: las palabras en la lengua mapuche, fundamentalmente las clases abiertas, experimentan ciertos procesos morfológicos y sintácticos que posibilitan el cambio de clase, ya sea por la intervención de algún sufijo derivacional, por la propiedad polisémica de algunas de las palabras, que pueden funcionar como clases distintas o por el cambio de posición en el orden de las palabras. Los procesos más productivos de formación de palabras suelen ser la composición y la derivación, no obstante, por razones de extensión, este artículo solo se concentra en la derivación.
\end{abstract}

Palabras clave: mapudungun, clases de palabras, derivación.

\section{The derivation and word class change in Mapudungun}

\begin{abstract}
This study examines the active and productive process of word classes changing in Mapudungun through derivation processes; that word formation process consists of a verbal lexeme, noun, adjective, or adverb linked to a derivational suffix. This is a descriptive study which follows the typological perspective of word class formation. The hypothesis establishes that words in mapuche language, mainly the open classes, under go certain morphological and syntactic processes that enable the change of word classes in either three ways: the intervention of some derivational suffix, polysemic properties of derivational suffixes, or the change of word order. While the most productive processes of word formation often are composition and derivation; due to length reasons this article will focus on the derivation process only.
\end{abstract}

Keywords: typology, word classes, derivation process.

Recibido: 05-05-2012

Aceptado: 26-11-2012

* Este estudio está vinculado al proyecto DICYT-USACH, No 031054LA, titulado ¿Cómo se forman las palabras en mapudungun?, año 2010-2011, Vicerrectoría de Investigación y Desarrollo de la USACH. Se agradece a la profesora Silvia Castillo, ayudante en este programa de investigación.

** Magíster en Lingüística. Universidad de Santiago de Chile, Santiago, Chile. elisa.loncon@usach.cl 


\section{Introducción}

En este estudio se analizan los principales tipos de derivaciones que cambian las clases de palabras en la lengua mapuche. Se podrá ver que las posibilidades de las derivaciones en esta lengua dependen en parte de su riqueza morfológica, en especial de la abundante cantidad de sufijos existentes y empleados en este proceso, y que entre sus propiedades manifiestan gran productividad, a la vez que son multifuncionales, sirven para derivar nuevas clases de palabras, como para marcar el aspecto, el modo y modificar las estructuras verbales, en Loncon (2011), se describen las características, propiedades y funcionamiento de las derivaciones que cambian las clases de palabras y entre ellas se presentan casos de verbalización, nominalización, adjetivación.

Existen diversos procedimientos de creación de palabras, en los que radica la posibilidad de ampliación léxica de una lengua. Según Chiodi y Loncon (1999), la derivación es el procedimiento más importante y de mayor uso en el Mapudungun. Este consiste en crear una nueva palabra derivándola de la combinación de un lexema raíz, un sustantivo, verbo, adjetivo y un sufijo.

La palabra mapudungun, nombre de la lengua mapuche, es una palabra compuesta que significa 'habla de la tierra', mapu significa 'tierra' y dungun 'habla'. Es hablada en Chile y Argentina, distinguiendo algunas variantes dialectales entre ambos países y también entre regiones en Chile, aunque estas variantes no dificultan la inteligibilidad o la comprensión de la lengua entre ellas, la mayoría de las variantes comparten la misma estructura gramatical.

Tipológicamente se caracteriza por ser una lengua aglutinante y polisintética (Augusta, 1903; Smeets, 2008, entre otros), que manifiesta el fenómeno de incorporación de objeto directo, y el rasgo pro drop, o de elisión del sujeto de la oración.

El Mapudungun es una lengua poco estudiada y en la actualidad son muy pocos los lingüistas dedicados a su investigación, por cuanto revelar argumentos que caracterizan el proceso derivacional del mapudungun, $\mathrm{o}$ de cualquier otra característica, contribuye a dar a conocer la lengua. El estudio también forma parte de las iniciativas destinadas a la preservación de la lengua, en el que la documentación de la misma cumple un papel 
importante, sobre todo cuando su existencia se encuentra amenazada por la sustitución del castellano.

\section{Antecedentes teóricos}

Desde un análisis tipológico, el mapudungun es una lengua aglutinante y polisintética (Salas, 1992; Zúñiga, 2006; Catrileo, 2010) que posee un patrón de incorporación de afijos que realizan cambios semánticos, morfológicos y sintácticos, incluyendo la incorporación de sustantivos, marca de objeto y de personas a la raíz verbal.

Según Aikenvald (2007), en las lenguas polisintéticas, una palabra puede estar constituida por varios morfemas con límites claramente definidos entre ellos, con una correspondencia de uno a uno entre el morfema y el significado, por lo que es de fácil segmentación e identificación. La autora también sostiene que, en la polisíntesis, hay elementos de gramaticalización de raíces independientes, y que estos elementos también pueden estar presentes en la formación de las palabras. Las propiedades señaladas se encuentran presentes en la lengua mapuche, y ayudan a comprender el fenómeno de la formación de palabras.

Por otro lado, existen diferentes tipos de derivaciones, según la lengua y la funcionalidad; así, hay derivaciones que cambian las clases de palabras, es decir, sustantivo $\leftrightarrow$ verbo; adjetivo $\longleftrightarrow$ sustantivo; adjetivo $\longleftrightarrow$ verbo. En la derivación, también ocurre cambio de valencia, generándose una subclase de verbos, tales como verbos intransitivos que cambian a transitivos, por ejemplo.

Con relación a la clase de palabra, se entiende por ello al grupo de palabras que realizan las mismas funciones sintácticas, que pertenecen a la misma clase de constituyentes; una clase se compone por formas que tienen las mismas funciones o posiciones en la frase. En las diferentes lenguas, las clases de palabras pueden ser abiertas o cerradas; las palabras abiertas corresponden a los sustantivos, verbos, adjetivos y adverbios, éstas se diferencian de las cerradas porque son susceptibles de aceptar nuevos miembros o palabras, ya sea a través de adiciones adquiridas en el tiempo, o por derivaciones de nuevas palabras (Dixon y Aikhenvald, 2006). Las clases cerradas, en cambio, no permiten la integración de nuevos elementos gramaticales, pues constituyen un número específico 
según sea la lengua; entre ellos se encuentran los artículos, preposiciones, conjunciones, interjecciones.

La palabra también es la unidad central de la interacción de la sintaxis con la morfología (Dixon, 2009). Sin embargo, en las lenguas particulares, algunas clases cerradas también manifiestan cambios a clases abiertas por acción de un derivacional, como se mostrará que ocurre en el mapudungun.

Según Catrileo (2010), en la lengua mapuche es un poco difícil determinar la clase de palabra porque tiene unidades léxicas especiales en donde la significación semántica del lexema individual depende de sus marcadores y del contexto en el cual ocurre el enunciado total. Las clases de palabras varían constantemente según la morfología derivacional y la función sintáctica. Catrileo (2010) al respecto señala que:

Gran parte del léxico del mapudungun está formado por un conjunto de lexemas y de raíces polivalentes que difícilmente pueden ser clasificadas como sustantivos, verbos, adjetivos y adverbios en forma aislada y fuera de contexto. (103)

Compartiendo la opinión de la cita, también es cierto y, como se demostrará más adelante, que la dificultad señalada, forma parte de la fortaleza de la lengua para la renovación lexical, porque permite distintas posibilidades de combinaciones entre clases abiertas y sufijos, para generar nuevas palabras.

Es destacable la propiedad de combinación de sufijos y raíces léxicas presentes en la lengua mapuche y la productividad de los sufijos derivacionales, como también ocurre con las construcciones atributivas.

Otro elemento importante en la tipología de la formación de palabras, es la gramaticalización, proceso empleado para estudiar los cambios producidos en la historia de la lengua y que afectan la morfología, sintaxis y contenido semántico de la palabra, aun cuando hay autores que sostienen que la gramaticalización conlleva a una pérdida de contenidos desde el punto de vista semántico. Al respecto, Bybee y Pagliuca (1985) sostienen que no hay tal desgaste semántico sino una generalización del significado, lo que provoca su polisemia y ambigüedad, y que hace posible el incremento de funciones adicionales. Estos cambios se expresan en la transformación de una palabra plena a un morfema, un afijo con funciones gramaticales que, al ligarse a palabras plenas, genera 
una nueva palabra y un nuevo significado, como ocurre en la lengua mapuche. Así, las palabras, aunque cambian en el tiempo, cumplen nuevas funciones lingüísticas y algunas conservan rasgos de su estado inicial (Bybee, 1998), los que portan e influyen en procesos sintácticos y morfológicos con el que actúan.

El mapudungun posee muchos sufijos derivativos y no todos cambian la clase de palabras. Los fenómenos ya descritos serán aplicados en el análisis de la lengua mapuche, a objeto de profundizar en qué consiste la derivación en esta lengua.

\section{Metodología}

En esta investigación, interesa describir procesos de derivación en la lengua mapuche a partir de datos empíricos del Mapudungun; por tal razón, el estudio se inscribe en el método descriptivo de la lingüística funcional y la corriente tipológica (Dixon, 2009). Para el análisis de las palabras, se ha realizado un registro del corpus de la lengua, en hablantes nativos de la Novena Región. Se grabaron textos orales, conversaciones y diferentes usos de la lengua (cuentos, cantos, historias, juegos, etc.). Las personas entrevistadas son adultos y ancianos, hombres y mujeres. Una vez registrado el corpus en audio, fue transcrito en la lengua y posteriormente, glosado morfológicamente.

El análisis morfológico de una palabra consiste en descomponerla en sus partes constituyentes hasta llegar a delimitar e identificar las unidades gramaticales mínimas. En este proceso, primero se segmentan las formas fonéticas de la palabra en unidades fónicas mínimas que portan un significado constante denominadas morfos. Se agrupan aquellos morfos que expresen un mismo significado como alomorfos de un mismo morfema, y finalmente se describen y se organizan de manera sistemática. La glosa contribuye al estudio del corpus y permite realizar diferentes tipos de relaciones sintácticas presentes en la frase, cláusulas y relaciones, características morfológicas entre las palabras.

Una vez glosados los datos, se procede a su análisis. Para ello, se contrastan frases bien formadas con otras mal formadas. Este procedimiento ayuda a dar cuenta de las restricciones que poseen los sufijos y los procesos derivativos; que, si bien funcionan con algunas estructuras, su uso no es posible en otras. Por ejemplo, la misma palabra kimeltuchekelu, para tener significado correcto, debe presentar el orden 
ya dado; en caso contrario, si invirtiéramos el orden y pusiéramos la raíz kimeltu al final, como en *chekelu-kimeltu, la frase se vuelve agramatical, no responde a la lógica de construcción en el Mapudungun; se trata de una palabra mal formada.

Los datos del corpus también han sido consultados en los diccionarios y libros de otros autores (Harmelink, 1996; Salas, 1992; Smeets, 2008).

Otro aspecto metodológico importante de destacar es el hecho de que su autora sea hablante nativa de la lengua mapuche, con conocimientos de la gramática de su lengua, condición que le permite contrastar información proporcionada por otros hablantes y, por ende, ofrecer un análisis más profundo del funcionamiento de la lengua. Ello no significa que los lingüistas no indígenas no aporten al estudio del mapudungun, sino enfatizar en la necesidad de que los nativos hablantes se involucren en el estudio de su propia lengua, y junto a la base lingüística disciplinar, aportar nuevas miradas al estudio de la misma.

\section{Presentación y análisis de los datos}

\subsubsection{El sufijo -tu}

El sufijo - $t u$ se liga a diferentes clases de palabras provocando cambios de la clase de palabra, en los tres primeros casos, de Sustantivo $\rightarrow$ verbo, adjetivo $\rightarrow$ verbo, adjetivo $\rightarrow$ sustantivo, sustantivo $\rightarrow$ adverbio, como se aprecia en el Cuadro $\mathrm{N}^{\circ} 1$ :

\section{Cuadro $\mathrm{N}^{\circ} \mathbf{1}$}

\begin{tabular}{|c|c|c|c|}
\hline $\mathrm{N} \rightarrow$ verbo & Adj $\rightarrow$ verbo & $\operatorname{adj} \rightarrow \mathbf{N}$ & $\mathrm{N} \rightarrow \mathrm{Adv}$ \\
\hline mamül $\rightarrow$ mamültu- & kelü $\rightarrow$ kelütu- & pichi $\rightarrow$ pichintun & manün $\rightarrow$ manüntu \\
\hline 'Leña' 'Buscar leña' & 'Rojo' 'Teñir de rojo"' & 'Chico' 'lo poco' & 'Pie' 'Andar a pie' \\
\hline kutral $\rightarrow$ kütraltu- & lif $\rightarrow$ liftu & afün $\rightarrow$ afütun & kürüf $\rightarrow$ kürüftu \\
\hline 'Fuego' 'Hacer fuego' & 'Limpio'_Limpiar' & 'Maduro' 'lo maduro' & 'viento' 'conviento' \\
\hline chadi $\rightarrow$ chaditu- & fücha $\rightarrow$ füchatu- & karü $\rightarrow$ karütu & liwentu $\rightarrow$ liwentu \\
\hline 'Sal"Salar la carne' & 'Malo' 'satisfacer' & 'Verde' 'comida cruda' & $\begin{array}{l}\text { 'Alba"en el } \\
\text { amanecer' }\end{array}$ \\
\hline poñü $\rightarrow$ poñitu- & weche $\rightarrow$ wechetu- & alu $\rightarrow$ alütu & ayen $\rightarrow$ ayentu \\
\hline 'Papa' 'Comer papa' & 'Joven' 'rejuvenecer' & 'Alü' 'Mucho tiempo' & 'risa' 'alegremente \\
\hline
\end{tabular}

El morfema - $t u$ es uno de los sufijos derivativos muy productivo 
de la lengua mapuche, cambia diferentes clases de palabras: verbos, sustantivos, adverbios, adjetivo.

$\mathrm{Al}$ analizar la primera columna a la izquierda, se observan sustantivos que cambian a verbos; entre ellos, mamültu- 'buscar leña', un típico verbo de recolección, que con el sufijo - $t u$ lexicaliza el objeto 'leña'; al igual que kütraltu- 'hacer fuego', lexicaliza el objeto 'fuego'. En este tipo, se encuentran todos los verbos derivados que impliquen hacer algo. Ocurre los mismo con el verbo de locación que también lexicaliza el objeto, por ejemplo, chaditunilo 'salar la carne'. En el caso de poñitun, 'comer papas', se trata de un verbo intransitivo, de ingestión, que como los anteriores lexicaliza el objeto afectado, es decir poñi, al que se le incorpora el rasgo ingerir, mediante el empleo del sufijo - $t u$. Así como este, hay muchos otros, como es el caso de matetun 'tomar mate', kofketun 'comer papas', korütün 'tomar sopa'.

En la segunda columna, el sufijo - $t u$, unido a adjetivo, también deriva verbos; como se observa en los ejemplos, $-t u$ desarrolla la noción indicada por el lexema adjetivo primario. En kelü-tu- $n$ 'tomar el color rojo', füchatun 'tomar la cualidad de viejo', indica probar o tomar la cualidad del adjetivo.

En la tercera columna, en el caso de los adjetivos que cambian a sustantivos, estos son de uso común y se refieren a palabras de tipo genérico, como decir, küpalimipichintu 'trajiste lo poco y nada'.

Finalmente, en la cuarta columna, se puede apreciar el uso adverbial de - $t u$, suele ser típico en frases de complemento, como namuntuküpay Pedro 'Pedro viene a pie', o Pedro küpaynamuntu. Kürüftumiawi 'andar tirando viento' o 'andar en la ventolera' liwentutripay 'salió temprano (de amanecida'), ayentunütranküley 'está conversando alegremente'.

Hay otros significados aportados por el sufijo -tu: acompañado de sustantivos referidos a lugares, tiene significado direccional, por ejemplo: 

(1) a. lewfu-tu-y
b. rüpü-tu-y
c. waria-tu-y
río-verb ${ }^{1}-2 \mathrm{~s}$
camino-verb-2s
'pueblo/villa-verb-2s
'Tomar la dirección del río' 'Tomar el camino'. 'Ir a la ciudad'.

\subsubsection{Los derivativos -nge y -le/küle $e^{2}$}

En el mapudungun se da una restricción equivalente a la que se observa en las construcciones atributivas, entre predicados con propiedades de nivel individual y realizado con el sufijo -nge, y las de nivel de estado que emplean el sufijo -le/-küle (Loncon, 2011). El contraste entre las propiedades que aportan los dos afijos del Mapudungun en las construcciones atributivas, es el siguiente:

-nge 'ser' $\rightarrow$ cópula $\rightarrow$ Predicados con propiedades a nivel individual

-le 'estar' $\rightarrow$ aspectual $\rightarrow$ Predicado con propiedades a nivel de estado.

Los morfemas -nge y-le/-küle también actúan como sufijos derivativos; pueden ligarse a adjetivos, sustantivos, verbos y pronombres, ejemplo: iñchengen 'yo soy', donde iñche es 'pronombre personal, primera persona'. Estos sufijos derivan diferentes tipos de verbos, -nge derivan verbos de propiedad y -le verbos estativos, como veremos:

(2)a. kümeche-nge-n

buena persona-AC-3s

'soy buena persona' a'. mañum-küle-y

agradecer-AA-3s

'está agradecida/o'

Los sufijos derivativos de propiedad corresponden a verbos resultantes de un lexema sustantivo ligado al morfema -nge; en este caso, el verbo resultante adquiere o tiene la propiedad del sustantivo, como se muestra en el Cuadro $\mathrm{N}^{\circ} 2$.

1 Adbreviatura: 1: primera persona; 2: segunda persona; 3: tercera persona; AA: afijo aspectual; AC: afijo copulativo; adj: adjetivo; adv: adverbio, AG: agentivo; Art: artículo; ASP: aspectual; d: dual; Det: determinante; DIR: direccional; FUT: futuro; GE: gerundio; HAB: habitual; NEG: negativo; p: plural; PART: participio; PERF: perfectivo; poss: posesivo; POSP: posposición; PL: pluralizador; s:singular; SD: sujeto dativo; v: verbo; VERB: verbalizador; *:frase agramatical.

2 Se usa -le después de una vocal, por ejemplo en amuley 'está yendo' y -küle, después de una consonante lefküley 'está corriendo'. Ambos sirven de nexo entre el sujeto y el predicado, y permiten marcas finitas (tiempo, número, y persona). 


\section{Cuadro $\mathrm{N}^{\circ}$ 2. Verbos derivados de -nge}

\begin{tabular}{|l|l|}
\hline Sustantivo $\rightarrow$ verbo & Adjetivo $\rightarrow$ verbo \\
\hline $\begin{array}{l}\tilde{n} u k e \rightarrow \tilde{n} u k e n g e-n \\
\text { 'madre' } \rightarrow \text { 'Ser madre' }\end{array}$ & $\begin{array}{l}\text { küllfün } \rightarrow \text { küllfü̈nge- } n \\
\text { 'bueno' } \rightarrow \text { 'ser ágil' }\end{array}$ \\
\hline $\begin{array}{l}\text { wirife } \rightarrow \text { wirifenge- } n \\
\text { 'Escritor' } \rightarrow \text { 'Ser escritor' }\end{array}$ & $\begin{array}{l}\text { fütra } \rightarrow \text { fütrange- } n \\
\text { 'grande' } \rightarrow \text { 'ser grande' }\end{array}$ \\
\hline
\end{tabular}

\section{Cuadro $N^{\circ} 3$. Derivados con -le/küle}

\begin{tabular}{|l|l|}
\hline Sustantivo $\rightarrow$ verbo & Adjetivo $\rightarrow$ verbo \\
\hline $\begin{array}{l}\text { wütreg } \rightarrow \text { wütregküley } \\
\text { 'noche' } \rightarrow \text { 'está de noche' }\end{array}$ & $\begin{array}{l}\text { poz } \rightarrow \text { pozküley } \\
\text { 'sucio' } \rightarrow \text { 'está sucio' }\end{array}$ \\
\hline $\begin{array}{l}\text { mawün } \rightarrow \text { mawünküley } \\
\text { 'Lluvia' } \rightarrow \text { 'está lloviendo' }\end{array}$ & $\begin{array}{l}\text { küme } \rightarrow \text { kümeley } \\
\text { 'bueno' } \rightarrow \text { 'esta bueno/a' }\end{array}$ \\
\hline
\end{tabular}

Como se observa en los ejemplos, los sustantivos y adjetivos que emplean el sufijo -nge marcan atributos permanentes y los sustantivos $\mathrm{y}$ adjetivos unidos a un sufijo -le/-küle marcan atributos transitorios. En el mapudungun, es muy frecuente encontrar adjetivos verbalizados; aunque hay menos que sustantivos; entre los adjetivos que funcionan de este modo, encontramos adjetivos referidos a clima, sabores, y de percepción.

\subsubsection{El sufijo -meke}

El sufijo -meke deriva verbos climáticos con palabras o sustantivos referidos al tiempo, como se aprecia a continuación:

\section{Cuadro $\mathrm{N}^{\circ} 4$}

\begin{tabular}{|l|l|l|}
\hline Sustantivo & Verbo & Significado \\
\hline Wün 'Amanecer' & Pün-meke-y & 'Anochece poco a poco' \\
\hline Kürüf 'Viento' & Kürüf-meke-ypukem. & 'Hay ventolera en invierno' \\
\hline
\end{tabular}

3 El sufijo -meke es homófono con el sufijo bimorfémico -me-ke, 'moverse frecuentemente hacia allá', empleado para indicar traslado frecuente de aquí hasta allá. 
Los ejemplos son casos de verbos referidos al clima con rasgos iterativos terminativos que aporta el sufijo -meke, en contraste con el sufijo -le/-küle que deriva eventos progresivos continuativos, como se puede ver en (3).

(3) a. Af -meke-y kachilla. terminar -PRG -3s trigo

'Acaba y acaba el trigo' b. Af -küle-y kachilla. terminar-AA -3 s trigo 'Se está acabando el trigo'.

El sufijo -meke también se puede emplear con algunos adjetivos, como se muestra:

\section{Cuadro $\mathrm{N}^{\circ} 5$}

\begin{tabular}{|l|l|l|}
\hline Adjetivo & Verbo & Adjetivos verbos significado \\
\hline küme 'bueno' & küme-meke- & 'hacerlo bien' \\
\hline weza 'malo' & wezameke- & 'hacerlo mal' \\
\hline kuru'negro' & 'kurümeke- & (No es aplicable a colores) \\
\hline
\end{tabular}

Como se puede observar, el morfema -meke ligado a un adjetivo no siempre es productivo con colores produce agramaticalidad.

\subsection{Otras propiedades de los morfemas -tu, -nge y -le/-küle}

Como se señaló al inicio, estos sufijos no solo intervienen en el cambio de clase de palabras, sino que además poseen propiedades que influyen en la sintaxis, y en la morfología, como veremos a continuación.

\subsubsection{Las propiedades sintácticas del sufijo $-t u$}

El sufijo - $t u$ es un dispositivo derivacional con muchas funciones. Autores anteriormente, a lo largo de los años, dieron cuenta de sus características, siendo Smeets quien entrega un esquema más completo de su funcionamiento, como se aprecia en el cuadro $\mathrm{N}^{\circ} 6$.

\section{Cuadro $\mathrm{N}^{\circ} 6$}

\begin{tabular}{|c|c|c|c|}
\hline \multicolumn{4}{|c|}{ Características del sufijo -tu según otros autores } \\
\hline Salas, 1992 & Harmelink, 1994 & Zúñiga, 2006 & Smeets, 2008 \\
\hline Repetitivo. & Verbalizador. & $\begin{array}{l}\text { De regreso. } \\
\text { Tiene variados } \\
\text { significados. }\end{array}$ & $\begin{array}{l}\text { Iterativo, } \\
\text { adverbializador, } \\
\text { Verbalizador, } \\
\text { transitivador. }\end{array}$ \\
\hline
\end{tabular}


Por otra parte, Aikhenvald (2011) señala que los dispositivos derivativos también pueden crear una subclase a través del cambio de valencias producido por su intervención, es decir, verbos intransitivos pueden adquirir la propiedad transitiva por la acción del derivativo. El sufijo- $t u$ perfectamente puede realizar este cambio de valencia, como se muestra en el ejemplo (4):

(4) a. Yafün 'Endurecer Yafu-tuami ilo 'Te servirás carne' b. Yafkün 'Enojarse' Yafkütuyñikarukatu 'Se enojó con su vecino'

El derivativo - $t u$ también puede verbalizar clases cerradas de palabras, como es el caso del interrogativo chum 'como':

(5) a. Chum-tu-ymi ;anay!?

Cómo-VERB-2s det, ¡Oye!

¿Qué te pasó ¡Oye!?

b. chempinge-y chem-a-y; feytakim-la- $n$.

Qué decir-3s que-FUT-3s; eso saber-NEG-1s.

'Qué le dijeron, tal vez; eso no lo supe'

De las palabras interrogativas mapuche, chum 'cómo' se diferencia de las otras, porque puede comportarse como un lexema verbal, no solo con el sufijo -tu, sino también con el sufijo -nge y -pe, como se observa:

(6) a. ¿Chum-pe -ymün?

Qué -PERF-2p

'¿Qué están haciendo?' b. ¿Chum-nge-lufemi-mi? qué -AC -PART hacer-2s

¿Por qué lo hiciste?'

c. chem-no-rumechem-la-iñ-mu, molesta-la-iñ-mu.

que NEG casi que-NEG-E1p-SD matestar-NEG-1pl-SD

'Nada nos hizo, no nos molestó',

Finalmente, a veces, el comportamiento de - $t u$, expuesto en los ejemplos anteriores, es poco predecible; tiene algunas propiedades aspectuales, se comporta como repetitivo, inversivo, reactivador de una acción determinada, como se observa a continuación: 
(7) a. Matuamu-tu-ymi mirukamew Adv ir-VERB-ASP-2s poss casa POSP

'Volviste a casa rápidamente' b. Poyera-tu-tu-y pu zomo waria mew Vestido-VERB-ASP-3s PL mujer 'Las mujeres se han vuelto a poner vestido'

En el ejemplo (7) a. el sufijo -tu es regresivo; el verbo original ir se convierte en regresar; en el segundo ejemplo(7) b., se presenta el sufijo $-t u$, dos veces, con la diferencia que en el primero la función se verbaliza el sustantivo, préstamo del español poyera, para formar poyeratutuy 'ponerse vestido'; y, en el segundo, - $t u$ marca el aspecto que indica ponerse vestido después de haber dejado de usarlo, es decir, restaura una acción que había dejado de realizarse.

\subsubsection{Sobre los otros usos de -nge}

Como se dijo anteriormente, el sufijo -nge, ligado a sustantivos y a adjetivos, forma verbos que expresan atributo de propiedad. A continuación, se proporcionan ejemplos referidos a cualidades físicas, psíquicas, morales, materiales, de origen o procedencia, posición, o pertenencia, o adscripción, que se forman con este sufijo derivativo:

(8) María trongli-nge-y.

'María es flaca'

$<$ Cualidad física $>$ María flaca $-\mathrm{AC}-3 \mathrm{~s}$

(9) [Künaruka]-nge-y.

$<$ Materia $>$ paja casa -AC-3s

(10) Pedro Santiaw-che -nge-y. 'Pedro es de Santiago' <Procedencia> Pedro Santiago -gente-AC -3s

(11) Niñukeñimapu-nge-y. mi madre su tierra $-\mathrm{AC}-3 \mathrm{~s}$

(12) Raúllawentu-che -fe-nge-y. 'Raúl es médico' 'La tierra es de mi madre' <Posesión>

Raul medicina-gente-AG-AC -3s

Raul medicina-gente-AG-AC-3s
$<$ Adscripción a una clase> 
(13) Rukü -nge-la -ya -ymi. 'No seas mezquino' mezquino-AC-NEG-FUT-2s
$<$ Valores $>$ $<$ Adj. percepción sensorial>

dulce -AC -3s Art manzana

\subsubsection{Los rasgos aspectuales de -le/-küle}

El sufijo -le/-küle se relaciona con el aspecto gramatical, corresponde al aspecto imperfectivo y se manifiesta cuando -le va acompañado de un verbo de actividad y de logro, fundamentalmente. No es compatible con verbos de estado, porque estos son:

+ estático, -télico y con rasgos -puntual, debido a sus propiedades aspectuales:

(15) Iñchiñ lef -küle-iñrüpümew.

Nosotros correr-AA -1p camino POSP

'Nosotros estamos corriendo en el camino'

(16) Trofkü -le -y wa kütral mew.

Reventar -AA -3s choclo fuego POSP

'Se está reventando el choclo en el fuego'

(17) *Lolo -le -iñ lepün mew.

hoyo-AA- $1 \mathrm{p}$ patio POSP

*no tiene traducción

Como se observa, el afijo -le con verbos de actividad, en (15) introduce predicados progresivos; en el ejemplo, se notan con mayor claridad las propiedades aspectuales de -le, similares al del auxiliar aspectual 'estar' del español en (16), el sufijo acompaña verbos de logro con el que también deriva eventos progresivos, continuativos. Estos son verbos dinámicos. El ejemplo 17 muestra una frase agramatical debido a que el sufijo no es compatible con verbos de realización. 


\subsubsection{El rasgo iterativo de -meke}

El sufijo -meke deriva eventos con rasgo ingresivo, intensivo y progresivo. Se diferencia de -le/-küle progresivo, porque -le es continuativo y -meke es iterativo. La presencia de dos progresivos en el Mapudungun había sido identificada anteriormente por Zúñiga (2000), quien los presentó como sinónimos, y también por Augusta (1903) y Salas (1992), aunque estos no profundizaron en su significado. En este análisis, se demuestra que -meke no es sinónimo de -le, a pesar de que ambos son progresivos.

El sufijo -meke no funciona con verbos estativos, debido a su naturaleza aspectual continuativa, como se observa en (18), en los siguientes ejemplos:
(18) a. * Kure -nge-meke-y.
b. *Nie-meke-y.
esposa-AC-hacer-3s tener-hacer-3s
'no tiene traducción' 'no tiene traducción'

Como se ha observado en los diferentes ejemplos de este análisis, la derivación depende de la intervención de ciertos sufijos que permiten cambio de clases de palabras, pero estos sufijos también generan temas verbales continuativos, atributivos y complejos. Al mismo tiempo, se puede decir que la elección de un verbalizador está determinada por la relación que establece la raíz léxica con el morfema derivativo; este último puede lexicalizar el agente, el objeto, el tema, dependiendo del tipo del verbo.

\subsection{El cambio de orden de las palabras}

En el mapudungun, no solo son importantes los morfemas derivacionales para identificar la clase de palabra, lo es también la ubicación de la palabra en relación al sustantivo y el verbo fundamentalmente, como se puede apreciar al analizar el ejemplo de Catrileo $(2010 ; 104)$ con la palabra KÜME.

Akuy Küme, ha llegado el buen tiempo'

Kümey, 'eso es bueno', 'está bien', 'tiene buen gusto'

Kümedungu, 'una buena noticia'

Kümetripay, 'resultó bien' 
En el ejemplo dado, observamos que una misma palabra cambia de clase según el contexto. A continuación, el siguiente cuadro muestra un ejemplo similar con otras palabras, cuyas clases cambian según la posición de la palabra en la frase respectiva.

Cuadro $N^{\circ} 7$ : cambios de clases de palabras

\begin{tabular}{|l|l|l|}
\hline Sustantivo & Verbo & Frase adjetiva \\
\hline $\begin{array}{l}\text { Mi zungun 'Tú } \\
\text { habla' }\end{array}$ & $\begin{array}{l}\text { Trawunmewzungun' Hable } \\
\text { en la reunión' }\end{array}$ & $\begin{array}{l}\text { Zugun che ' Habla de } \\
\text { la gente' }\end{array}$ \\
\hline $\begin{array}{l}\text { Wetrekan' Paso } \\
\text { nuevo' }\end{array}$ & $\begin{array}{l}\text { Epuantütrekan 'Camine } \\
\text { dos días' }\end{array}$ & $\begin{array}{l}\text { Trekanwaka 'Pasos de } \\
\text { vaca' }\end{array}$ \\
\hline Ngüman 'Llanto' & $\begin{array}{l}\text { Ngüman mi amutun 'Lloré } \\
\text { tu partida' }\end{array}$ & $\begin{array}{l}\text { Ngümandungu } \\
\text { 'Asuntos de tristeza' }\end{array}$ \\
\hline
\end{tabular}

En el Cuadro 7, todas las palabras emplean el mismo sufijo - $n$, este como los anteriores es un sufijo derivacional (Smeets, 2008), con funciones múltiples, y puede formar sustantivos, verbos o adjetivos.

Como se ha analizado, la clase de palabra depende de la función de la misma en la frase y según el orden que ocupa, puede ser un sintagma nominal, verbal o adjetival. Dicho lo anterior, y para comprender el cambio de clase de palabras en la lengua mapuche, hay que considerar diferentes criterios de análisis, en los que intervienen los semánticos, pero también los rasgos morfológicos y sintácticos que aportan los sufijos derivativos. De igual modo, no basta conocer el rasgo semántico atribuido a la palabra derivada, sino la función que cumple el sufijo derivativo al unirse a una determinada palabra, o la posición de la palabra en la frase o el sintagma.

\section{Conclusiones}

Como se ha analizado, la clase de palabra depende de la función de la misma en la frase y, según el orden que ocupa, puede ser un sintagma nominal, verbal o adjetival. Dicho lo anterior, y para comprender el cambio de clase de palabras en la lengua mapuche, hay que considerar diferentes criterios de análisis, en los que intervienen los semánticos, pero también los rasgos morfológicos y sintácticos que aportan los sufijos derivativos. De igual modo, no basta conocer el rasgo semántico atribuido a la palabra derivada, sino la función que cumple el sufijo derivativo al 
unirse a una determinada palabra, o la posición de la palabra en la frase o el sintagma.

Los sufijos derivativos del mapudungun son altamente productivos en el procedimiento de derivación y comparten, entre ellos, una serie de propiedades morfológicas y léxicas, transforman verbos adjetivos y adverbios, en diferentes clases de palabras, siendo el sufijo - $t u$ uno de los que más funciones realiza. Por otro lado, las clases de palabras varían constantemente según la morfología derivacional y la función sintáctica, esta flexibilidad de la clase de palabra es fundamental para comprender la polisemia de las palabras y la productividad de los sufijos derivativos.

Es importante señalar que los sufijos que intervienen en la derivación toman como material de entrada raíces nominales, adjetivales o verbales. La explicación de este tipo de comportamiento lingüístico es -sin lugar a dudas- de carácter teórico, ya que depende -por un lado- de la definición de clases de palabras que asumamos y -por otro- de las propuestas de la morfología derivativa. Este comportamiento de las raíces léxicas del mapudungun impide el poder considerar que exista una clara definición de clases de palabras, al menos al nivel del léxico.

Finalmente, las propiedades estructurales del mapudungun, sus rasgos tipológicos en tanto lengua aglutinante y polisintética, la relativa flexibilidad en el orden de palabras influye directamente en la productividad de la derivación y en el cambio de clases de palabras.

\section{Bibliografía}

Aikhenvald, A. (2007). Typological dimensions in word-formation. En Language Typology and Syntactic Description. Grammatical Categories and the Lexicon. Cambridge, UK: Cambridge University Press: 1-65.

y R. Dixon (2011). Language at large. Leiden: Brill.

Augusta, F. de (1903). Gramática Mapuche Bilingüe. Santiago de Chile: Ediciones Séneca.

(2007). (Ed.). Diccionario Araucano-Español y Español-Araucano ( $4^{\mathrm{a}}$ ed.).Santiago de Chile: Cerro Manquehue.

Bybee, J. (1998). The Emergent Lexicon.CLS 34: The Panels. University of Chicago: Chicago Linguistic Society: 421-435.

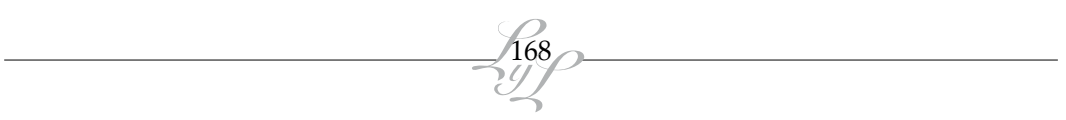


Bybee, J. \& W. Paliuca (1985). Cross-lingüistic comparison and the development of grammatical meaning. Fisiak, J.(Ed.).Historical Semnatics and Historical Word Formation. Berlin: Mouton: 59-83.

Catrileo, M. (2010). La Lengua Mapuche en el Siglo XXI. Valdivia: Universidad Austral de Chile.

Company, C. (2004). La gramaticalización por subjetivización como prescindibilidad de la sintaxis en Nueva Revista de Filología Hispánica, México: El Colegio de México: $1-27$.

Cormier, B. (1981). Language Universals and Linguistic Typology. Chicago: University of Chicago Press.

Chiodi, F. y E. Loncon (1999). Crear nuevas palabras, innovación y expansión de los recursos lexicales del Mapuzugun. Temuco: UFRO, Instituto de Estudios Indígenas y CONADI unidad de cultura y educación.

Dixon, R. (2009). Basic Linguistic Theory. Oxford: Oxford University Press.

\& A. Aikhenvald (2006). Complementation. Oxford: Oxford University Press.

Estrada, Z. (2005). Clases verbales en el Pima Bajo. En Memorias del Congreso de Idiomas Indígenas de LatinoaméricaII, 27 -29 de octubre del 2005. University of Texas at Austin, en <http://www.ailla.utexas.org/site/ cilla2/Estrada_CILLA2_pimabajo.pdf>

Loncon, E. (2011). Morfología y aspectos del mapudungun. México: Biblioteca de Signos Universidad Autónoma Metropolitana, Unidad Iztapalapa.

(2007). Propiedades de los afijos -nge y -le del Mapudungun. Revista Anales de Antropología. Vol. 41. Instituto de Investigación Antropológica, UNAM: 60-82.

Salas, A. (1992). El Mapuche o Araucano. Valparaíso: Editorial Mapfre.

Smeets, I. (2008). A Grammar of Mapuche. Berlín: Mouton de Gruyter. 
Zúñiga, F. (2006). Mapudungun. El Habla Mapuche. Santiago de Chile: Centro de Estudios Públicos.

(2001). Dos Progresivos y dos Regulativos en Mapudungun. En LIAMES. Brazil: Instituto de Estudios da Linguagem, Departamento de Lingüística, UNICAM: 63-75. 